\title{
CYSTINOSIS FOUNDATION SYMPOSIUM
}

\author{
Tuesday, April 28, 1987 \\ AVALON A \& B
}

(Hotel Convention Center)

6:30 P.M.

Update on the Collaborative Cysteamine Study

Experience with Carnitine Therapy

Cysteamine Study Update

Future Directions
Dr. William Gahl

$\mathrm{NIH}$

Dr. Jess G. Thoene

University of Michigan

Dr. Jerry A. Schneider

University of California, San Diego

$7: 30$ P.M.

Hors d'oeuvres and no host bar reception 